\title{
Correspondence
}

\section{The LMA for unplanned prolonged procedures}

To the Editor:

Asai and Morris suggest that the laryngeal mask airway (LMA) should not be used for procedures lasting $>2$ hr. ${ }^{1}$ Their reasoning focuses on the theoretical risk of increased pharyngeal morbidity and regurgitation. However, procedures frequently take longer than predicted and the question arises as to whether the LMA should be removed and the trachea intubated if anaesthesia extends beyond two hours. We would like to present data from 15 patients who underwent planned prolonged anaesthesia which suggests that in the hands of experienced personnel the technique is safe for some procedures of 4-8 hr duration. All patients were ASA 1-3 and underwent lower limb orthopaedic or plastic surgery. A balanced regional technique was utilised as previously described. ${ }^{2}$ The mean (range) for age, weight and procedure duration was 36 (18-52) yr, 81 (57-92) kg and 4.6 (4.1-7.8) hrs. All LMA insertions were successful at the first attempt with a median fibreoptic score of 3.3. The $\mathrm{SpO}_{2}$ remained $>95 \%$ and $\mathrm{PETCO}_{2}$ ranged from 34 to $68 \mathrm{mmHg}$. In four patients, thoraco-abdominal movement was monitored with two extensometers ${ }^{2}$ and there was no evidence of respiratory fatigue. There was also no evidence of positional instability of the LMA cuff or regurgitation. Three patients developed a mild sore throat, but there were no other adverse sequelae. These data lend further support to the concept that prolonged LMA usage is safe $^{2,3}$ and suggests that there is no need to exchange the LMA for a tracheal tube if surgery unexpectedly exceeds two hours.

\section{J. Brimacombe MB ChB FRCA \\ J. Archdeacon MB BS FANZCA \\ Cairns Base Hospital \\ The Esplanade \\ Cairns 4870 \\ Australia}

\section{REFERENCES}

1 Asai $T$, Morris $S$. The laryngeal mask airway: its features, effects and role. Can J Anaesth 1994; 41: 930-60.

2 Brimacombe J, Shorney $N$. The laryngeal mask airway and prolonged balanced anaesthesia. Can J Anaesth 1993; 40: 360-4.
3 Verghese C, Brimacombe J. Survey of laryngeal mask usage in 11910 patients - safety and efficacy for conventional and nonconventional usage. Anesth Analg (in press).

\section{REPLY}

In our review article, we did not suggest that the CMA should not be used for procedures lasting greater than two hours, and did not discuss whether the laryngeal mask should then be removed and the trachea intubated. We stated that "it is not clear whether the incidence of regurgitation increases with the duration of surgery when the laryngeal mask is used." We also suggested that "it is not possible to define how long the airway can be safely managed with the laryngeal mask, but continuous vigilance is required during its use."I

A large number of patients are required to show that the incidence of pulmonary aspiration does not increase with prolonged use of the laryngeal mask. Drs. Brimacombe and Berry estimated the incidence of pulmonary aspiration for which intensive care is required after the use of the laryngeal mask is between 1:900 and 1: 250,000. ${ }^{2}$ To show that the incidence is no greater than this with prolonged use of the laryngeal mask, between 30,000 and 750,000 patients would be required. ${ }^{3}$

We believe that our statement from the review article is sensible and would be supported by a majority of anaesthetists. "It is prudent not to use the laryngeal mask for prolonged anaesthesia until controlled studies show that this is safe" (authors italics).

Takashi Asai MD

Stephen Morris FRCA

Department of Anaesthetics and Intensive Care Medicine

University of Wales College of Medicine

Heath Park, Cardiff, CF4 4XN

United Kingdom

\section{REFERENCES}

1 Asai $T$, Morris $S$. The laryngeal mask airway: its features, effects and role. Can J Anaesth 1994; 41: 930-60.

2 Brimacombe J, Berry A. Aspiration and the laryngeal mask airway - a survey of Australian intensive care units (Letter). Anaesth Intensive Care 1992; 20: 534-5.

3 Hanley $J A$, Lippman-Hand $A$. If nothing goes wrong, is everything all right? JAMA 1983; 249: 1743-5.

\section{The LMA in intracranial aneurysm surgery}

To the Editor:

The anaesthetic management of intracranial aneurysms is a trade-off in avoiding hypertension to prevent aneurysmal rerupture ${ }^{1}$ and hypotension to prevent aggravation of pre-existing cerebral ischaemia due to vasospasm. ${ }^{2-4}$ There are several advantages of the laryngeal mask air- 